\title{
Tre osservazioni su Diez tesis ACERCA DE LA COHERENCIA EN EL DERECHO
}

Aldo Schiavello

1. Premessa

Come mette in luce sin dall'inizio del suo saggio anche Amalia Amaya, il tema della coerenza (coherence) occupa un posto importante nel dibattito filosofico contemporaneo. Ciò è dovuto a molteplici ragioni solo in parte convergenti.

In relazione alla filosofia teoretica ed alla epistemologia va rilevata la sostituzione di un modello di conoscenza di tipo descrittivistico ${ }^{1}$, peculiare del neo-positivismo più intransigente, con un modello olistico

${ }^{1}$ Con il termine 'descrittivismo' si intende comunemente quella concezione epistemologica secondo la quale il compito del linguaggio della conoscenza è quello di

Revista Discusiones X - 155 
che mette in evidenza la natura sistematica dell' attività conoscitiva e, di conseguenza, l'impossibilità di predicare la verità o la falsità del singolo enunciato, ma solo del sistema complessivo delle credenze e convinzioni razionali ${ }^{2}$. L'olismo intende la conoscenza come «il rapporto globale fra uno schema concettuale e un campo di esperienza ${ }^{3}$. Joseph Raz rileva l'esistenza di un rapporto stringente, anche se non di carattere logico ${ }^{4}$, tra olismo e coerenza, quasi si trattasse delle stesse tesi espresse da prospettive diverse ${ }^{5}$. Egli definisce infatti l'olismo come «[...] la tesi secondo cui ogni cosa è collegata ad ogni altra cosa»e, a partire da questa definizione, osserva che: «se ogni cosa è collegata ad ogni altra

rappresentare il mondo "così com'è". Si può dire -per usare una ben nota metafora di Richard Rorty- che i descrittivisti ritengono che la mente umana funzioni come uno specchio capace di "rispecchiare la realtà". Cfr. R. Rorty, La filosofia e lo specchio della natura (1980), trad. it. G. Millone e R. Salizzoni, Bompiani, Milano, 1986, p. 12 e V. Villa, Il positivismo giuridico: metodi, teorie e giudizi di valore. Lezioni di filosofia del diritto, Giappichelli, Torino, 2004, pp. 141156.

${ }^{2}$ Sull'olismo, cui qui si è inteso semplicemente accennare, si vedano: P. Duhem, La teoria fisica: il suo oggetto e la sua struttura (1906), Il Mulino, Bologna 1978; W. V. O. Quine, Due dogmi dell'empirismo (1951), in A. Pasquinelli (a cura di), Il neoempirismo, Utet, Torino 1969; W. V. O. Quine, Parola e oggetto (1960), trad. it. di F. Mondadori, Il Saggiatore, Milano 1970; W. V. O. Quine, Il problema del significato (1953), trad. it. di E. Mistretta, Ubaldini, Roma 1966; A. G. Gargani, Lo stupore e il caso, Laterza, Roma-Bari 1985.

${ }^{3}$ V. Villa, Conoscenza giuridica e concetto di diritto positivo, Giappichelli, Torino 1993, p. 203.

${ }^{4}$ Andrei Marmor a questo proposito osserva che: «l'olismo [...] non implica una teoria coerentista. Si tratta di una prospettiva negativa, nel senso che non offre una risposta alla questione su come rimpiazzare il fondazionalismo, e una teoria coerentista della conoscenza è solo uno dei possibili candidati a ricoprire questo ruolo» [A. Marmor, Interpretation and Legal Theory, Clarendon Press, Oxford 1992, p. 79].

${ }^{5}$ Si veda J. Raz, The Relevance of Coherence, in J. Raz, Ethics in the Public DomainEssays in the Morality of Law and Politics, Clarendon Press, Oxford 1994, p. 283, n. 11. Cfr. anche J. M. Pérez Bermejo, Alcune osservazioni sul valore della coerenza nei sistemi giuridici, "Diritto e questioni pubbliche" (www.dirittoequestionipubbliche.org), n. 7, 2007, pp. 43-59, in particolare pp. 51-56. 
cosa, come è possibile distinguere tra verità e falsità se non mediante un test di coerenza?» ${ }^{6}$

In relazione alla filosofia pratica, è evidente l'influenza della teoria di John Rawls, ormai da decenni al centro del dibattito sull'etica. In particolare, per quel che riguarda il tema della coerenza è opportuno ricordare la concezione rawlsiana dell'equilibrio riflessivo, secondo la quale le nostre intuizioni morali debbono passare al vaglio di principi generali o, addirittura, di una compiuta teoria morale. Tale procedura permette di adattare le nostre intuizioni ad una teoria morale coerente. Quest'ultima, a sua volta, può subire delle modifiche se si dimostra incapace di giustificare alcune intuizioni particolarmente importanti. Il risultato di questo processo è una situazione di tendenziale equilibrio in cui intuizioni e teoria morale si bilanciano a vicenda. Rawls sottolinea che questo esito non va considerato come una conquista definitiva in quanto, dopo aver raggiunto una posizione di equilibrio, si passa ad uno stadio successivo di instabilità che prelude ad un equilibrio ulteriore, e così all'infinito ${ }^{7}$.

L'olismo e l'equilibrio riflessivo -pur essendo spesso indicati in coppia come ragioni di sfondo dell'interesse per la coerenza nel dibattito filosofico generale- non si implicano necessariamente tra loro: è plausibile accogliere una posizione convenzionalistico-olistica in campo epistemologico e, ad esempio, aderire ad una prospettiva anticognitivista in etica o accogliere l'equilibrio riflessivo a partire da una concezione neo-positivistica della conoscenza. Un esempio significativo di quest' ultima possibilità è rappresentato dallo stesso Rawls. Egli, pur

${ }^{6}$ J. Raz, The Relevance of Coherence, cit., p. 278. Esistono tuttavia concezioni dell'olismo più deboli rispetto a quella ricostruita da Raz. Cfr. M. Iglesias Vila, El problema de la discreción judicial. Una aproximación al conocimiento jurídico, Centro de Estudios Políticos y Constitucionales, Madrid, 1999, pp. 215-217; G. Maniaci, Razionalità ed equilibrio riflessivo nell'argomentazione giudiziale, Giappichelli, Torino, 2008, pp. 219-220.

${ }^{7}$ Cfr. J. Rawls, Una teoria della giustizia (1971), trad. it. di U. Santini riv. da S. Maffettone, Feltrinelli, Milano 1984, pp. 32-36 e J. Rawls, Liberalismo Politico (1993), trad. it. G. Rigamonti, Edizioni di Comunità, Milano, 1994, p. 42. Sulla relazione tra equilibrio riflessivo e coerenza, con particolare riguardo all'argomentazione giudiziale, cfr. G. Maniaci, Razionalità ed equilibrio riflessivo nell'argomentazione giudiziale, cit., pp. 231-326. 
sottolineando che l'equilibrio riflessivo costituisce una procedura argomentativa che travalica l'ambito dell'etica ${ }^{8}$, tende a marcare fortemente la differenza tra scienze sociali e scienze naturali ${ }^{9}$, e ciò evidentemente non è conciliabile con una concezione costruttivistica e coerentista della conoscenza ${ }^{10}$. Questa constatazione, tuttavia, può essere messa tra parentesi in quanto non inficia il fatto che la coerenza ricopre un ruolo rilevante nella riflessione filosofica contemporanea.

Anche nell'ambito più ristretto del diritto, della filosofia del diritto e della scienza giuridica è possibile individuare alcuni fattori specifici che, a partire dagli ultimi decenni del secolo scorso, hanno contribuito a produrre un rinnovato interesse per il tema della coerenza ${ }^{11}$.

${ }^{8}$ Rawls, ad esempio, cita sia Nelson Goodman che utilizza uno schema argomentativo simile al suo per spiegare l'inferenza induttiva e deduttiva, sia Joel Feinberg che paragona il metodo argomentativo della discussione filosofica alle discussioni nelle corti di giustizia dove continuamente si aggiustano fra loro principi e precedenti giudiziari. Cfr. N. Goodman, Fact, Fiction and Forecast, Bobbs-Merrill, Indianapolis, 1973, in particolare p. 64; J. Feinberg, Social Philosophy, Englewood Cliffs, New York 1973, in particolare p. 34 .

${ }^{9}$ Rawls, dopo avere presentato il ruolo che l'equilibrio riflessivo gioca in filosofia morale, scrive: «Lo stesso non vale ad esempio per la fisica. Prendiamo un caso limite: se siamo in possesso di una accurata descrizione del moto dei corpi celesti che non è di nostro gradimento, non ci è dato di alterare questi moti per renderli compatibili con una teoria più attraente. Il fatto che i principi della meccanica celeste possiedono una loro bellezza intellettuale è dovuto soltanto a un caso fortuito» [J. Rawls, Una teoria della giustizia, cit., p. 57]. Sembra evidente, alla luce di questa citazione, che in ambito epistemologico Rawls accoglie una posizione realistica particolarmente ingenua, se non addirittura una concezione baconiana della conoscenza. Cfr. l'interessante M. Hanen, Justification as Coherence, in M. A. Stewart (ed.), Law, Morality, and Rights, Reidel, Dordrecht, 1983, pp. 67-92.

${ }^{10}$ Si utilizza il termine costruttivismo post-analitico per indicare quella prospettiva epistemologica che può farsi risalire alle riflessioni del secondo Wittgenstein. Per una compiuta presentazione di questa concezione filosofica si veda V. Villa, Conoscenza giuridica e concetto di diritto positivo, cit., pp. 187228.

${ }^{11}$ Seguo qui G. Pino, Coerenza e verità nell'argomentazione giuridica. Alcune riflessioni, "Rivista internazionale di filosofia del diritto", IV serie, LXXV, 1, 1998, pp. 84-126, in particolare pp. 84-90. 
Tra questi fattori è opportuno menzionare il fiorire di teorie dell'argomentazione giuridica, la centralità sempre più evidente dei principi- e di quelli costituzionali in particolare -nell'argomentazione giudiziale e dottrinaria, il passaggio da teorie del diritto oggettualistiche a teorie del diritto come pratica sociale ${ }^{12}$, la complessità dei sistemi giuridici contemporanei che sfugge alle teorie tradizionali del sistema giuridico e delle fonti del diritto. Ė appena il caso di precisare che anche in precedenza la coerenza ricopriva un ruolo nelle riflessioni dei giusfilosofi e dei giuristi, ma si trattava di un ruolo marginale, collegato alla eliminazione di contraddizioni logiche tra i materiali normativi di un sistema giuridico ${ }^{13}$.

La ricerca di Amaya sul ruolo della coerenza nel diritto ha un duplice pregio. Il primo -collegato in qualche modo a queste riflessioni introduttive- consiste nel fatto che la concezione della coerenza in ambito giuridico tratteggiata in Diez tesis acerca de la coherencia en el derecho (da ora in poi, Diez tesis) è fondata su una serie di assunti filosofico-generali che contribuiscono alla solidità della teoria. Non di rado, gli studi su coerenza e diritto mostrano una elevata impermeabilità a contaminazioni di tipo filosofico (per non parlare di quelle derivate dalla psicologia); ciò spesso non è dovuto a trascuratezza, ma è collegato alla convinzione dei giuristi che la pratica giuridica presenti una specificità tale da rendere superfluo, se non addirittura controproducente, lo sforzo di inserire le riflessioni su diritto e coerenza in un quadro più generale. Pur non potendo approfondire questo punto, ritengo che tale convinzione sia tanto più difficile da sostenere quanto più la coerenza acquisisca un ruolo centrale e pervasivo nel diritto.

Il secondo pregio è quello di trattare in modo unitario, fatte salve alcune differenze, la coerenza normativa, che riguarda la giustificazione di proposizioni normative, e la coerenza fattuale o narrativa, che riguarda la prova dei fatti. L'idea di proporre una concezione unitaria della giustificazione giuridica mi sembra al tempo

${ }^{12}$ Cfr. F. Viola, G. Zaccaria, Diritto e interpretazione. Lineamenti di teoria ermeneutica del diritto, Laterza, Roma-Bari, 1999, pp. 21-44; F. Viola, Il diritto come pratica sociale, Edizioni Universitarie Jaca, Milano, 1990.

${ }^{13}$ Cfr. ad esempio N. Bobbio, Teoria generale del diritto, Giappichelli, Torino, 1993, pp. 201-235. 
stesso convincente ed originale. Originale perché di solito la teoria del ragionamento giuridico si occupa esclusivamente della coerenza normativa mentre la teoria della prova della coerenza fattuale; convincente in quanto mi sembra che una concezione unitaria della coerenza si inserisca bene all'interno del paradigma del diritto come pratica sociale, a cui abbiamo accennato appena sopra, che si caratterizza proprio per l'ambizione di collegare in modo dinamico fatti e norme ${ }^{14}$.

I prossimi paragrafi sono dedicati ad approfondire tre osservazioni critiche sulla concezione della coerenza difesa da Amaya. Tali osservazioni riguardano: a) la presunta incompatibilità tra coerenza e concezione strumentale della ragion pratica; b) l'aspirazione alla best explanation e, infine, c) il collegamento tra coerenza e responsabilità epistemica.

Prima di procedere, è però opportuno esplicitare il contesto ideologico ${ }^{15}$ all'interno del quale si situano queste obiezioni. Condivido infatti la convinzione di Uberto Scarpelli secondo il quale, nell' ambito

${ }^{14}$ Questo modello è ben rappresentato dalla practice theory of norms di Herbert Hart. In breve, attraverso questa teoria Hart affronta in modo più convincente rispetto ad Hans Kelsen il problema del fondamento della validità delle norme giuridiche: una regola è una regola giuridica valida se soddisfa i criteri stabiliti dalla regola di riconoscimento, che, a differenza della norma fondamentale, è una regola sociale. La regola di riconoscimento è il criterio supremo (la "regola delle regole") che consente di ricondurre ad unità sistemica tutte le regole giuridiche e di distinguere queste ultime dalle regole morali o dalle regole di etichetta. In ultima istanza, dunque, il diritto poggia su una pratica sociale e ciò spiega il legame inscindibile tra fatti e norme.

${ }^{15}$ Norberto Bobbio distingue un senso debole ed un senso forte di 'ideologia'. Nella prima accezione, 'ideologia' indica un insieme di idee condivise che guidano i comportamenti politici; nella seconda, indica forme di mistificazione, (auto)illusione e falsa coscienza. Il senso in cui nel testo si parla di 'contesto ideologico' è riconducibile all' accezione debole di 'ideologia'. Cfr. N. Bobbio, L'ideologia in Pareto e in Marx (1968), ora in Id., Saggi sulla scienza politica in Italia, Laterza, Bari, 1971, pp. 109-122. La mia attenzione su questa distinzione e sul saggio di Bobbio è stata richiamata da E. Diciotti, Regola di riconoscimento e concezione retorica del diritto, "Diritto e questioni pubbliche" (www.dirittoequestionipubbliche.org), 7, 2007, pp. 9-42, in particolare p. 38, n. 35 . 
delle scienze umane, il massimo di scientificità possibile è garantito dalla esplicitazione dei propri assunti valoriali e non certo da una improbabile neutralità dello studioso ${ }^{16}$.

Personalmente ritengo che l'atteggiamento più adeguato rispetto alle teorie coerentiste del diritto sia rappresentato dalla diffidenza. Ciò impone di adottare, nei confronti di tali teorie, la pratica del sospetto come appropriato metodo filosofico. Ad esempio, il Trasimaco di Platone afferma: «la giustizia è [...] l'utile del più forte...» ${ }^{17}$. Il merito della tesi di Trasimaco è quello di mostrare la differenza tra un'apparenza e la realtà. L'apparenza è che ogni proposta di giustizia è universale e, dunque, vale per tutti coloro che ricadono nel suo ambito di applicazione; la realtà è che la giustizia riguarda soltanto i più forti e la tutela del loro utile. Tuttavia, l'aspetto più interessante della tesi di Trasimaco non è la "realtà", vera o presunta, che essa ci comunica, ma l'aver avanzato il sospetto che quel che appare non corrisponda necessariamente a ciò che $\mathrm{e}^{18}$. Essa solleva un velo senza poterci dire cosa veramente esso nasconda o, meglio, suggerisce che $\mathrm{i}$ criteri di giustizia possono svolgere un ruolo di copertura. La "pratica della diffidenza" deve quindi essere intesa come un invito a prendere sul serio la ragionevolezza e l'argomentazione e ad evitare di trasformarle in artifici retorici, attraverso i quali soffocare sul nascere, piuttosto che comporre, i disaccordi ed i conflitti che rappresentano una caratteristica essenziale delle società pluraliste e multiculturali.

Che le teorie coerentiste spaccino sotto le (mentite) spoglie di una attraente teoria del sistema giuridico una politica del diritto discutibile è un sospetto che merita di essere preso sul serio. Come sostiene in particolare il realismo giuridico, potrebbe darsi che le teorie coerentiste amplino surrettiziamente la discrezionalità dell'interprete e del giudice in particolare affermando di voler fare esattamente il

${ }^{16}$ Cfr. U. Scarpelli, Il metodo giuridico (1971), ora in Id., L'etica senza verità, Il Mulino, Bologna, 1982, pp. 179-203.

${ }^{17}$ Platone, La Repubblica, trad. it. M. Vitali, Feltrinelli, Milano, 1995, 338c-d.

${ }^{18}$ Riprendo questa interpretazione delle tesi del Trasimaco di Platone da A M. Iacono, La giustizia di Trasimaco e i filosofi del sospetto, "Ragion Pratica”, 20, 2003, pp. 167-185. 
contrario $^{19}$. Questo è l'aspetto più marcatamente ideologico e retorico delle teorie coerentiste che è compito della scienza giuridica tentare di svelare. Le osservazioni che seguono sul saggio di Amaya sono tutte accomunate da questo intento.

È corretto riconoscere, a conclusione di questa premessa, che la stessa Amaya non concede "sconti" particolari al coerentismo giuridico né indulge alla tentazione di oscurare le difficoltà che tale prospettiva deve fronteggiare.

\section{Coerenza E CONCEZIOne StRumentale Della RAgion PRATICA}

Secondo Amaya, il ragionamento coerentista è incompatibile con una concezione strumentale della ragion pratica. Tale concezione «...es problemátic[a] en cuanto que sitúa los fines y valores fuera del ámbito de la razón, como si fueran puntos firmes, fijados meramente por las preferencias individuales, y no revisables racionalmente, que constriñen el espacio de la deliberación» ${ }^{20}$. Visto poi che i fini perseguiti dal diritto sono molteplici e spesso in conflitto, una concezione strumentale della ragion pratica, non avendo nulla da dire sulla risoluzione di questi conflitti, risulta particolarmente insoddisfacente.

La tesi della incompatibilità tra concezione strumentale della ragion pratica e ragionamento coerentista è molto ambiziosa e, a mio avviso, sbagliata.

Innanzitutto, e come semplice constatazione, esistono alcune teorie coerentiste che presuppongono una concezione internalista delle ragioni. Un esempio significativo è rappresentato dalla teoria del ragionamento giuridico di Neil MacCormick: si può riconoscere un ruolo importante alla coerenza in ambito giuridico e, al tempo stesso, ammettere che, in alcuni casi, esistono genuini "disaccordi pratici" che

\footnotetext{
${ }^{19}$ Per una ottima presentazione di queste critiche, cfr. G. B. Ratti, Norme, principi e logica, Aracne, Roma, 2009, pp. 77-89.

${ }^{20}$ A. Amaya, Diez tesis, p. 44.

162 - Revista Discusiones X
} 
non possono essere composti attraverso la ragione ${ }^{21}$. Ciò significa, in altri termini, che non sempre esiste un'unica soluzione corretta, anche se, attraverso la ragione, è sempre possibile escludere un certo numero di soluzioni sbagliate. Personalmente, ritengo che il conflitto sia un elemento insopprimibile della pratica giuridica (a maggior ragione in società pluraliste e multiculturali) e che compito del giurista, del filosofo del diritto, sia quello di individuare i conflitti genuini e non componibili e non quello di nasconderli.

Amaya non è insensibile al problema del conflitto tra ragioni. Ella riprende infatti la proposta di Susan Hurley la quale ritiene che «las razones para la acción son razones pro tanto, que entran en conflicto genuino y que retienen una fuerza residual. Por ejemplo, es bien posible que un acto sea justo pero inclemente, y que ese acto sea correcto, en cuanto que es el que favorece la teoría acerca de la justicia y la caridad más coherente que se ha podido construir en el curso de la deliberación» ${ }^{22}$. Il presupposto di questa prospettiva è tuttavia che sia possibile individuare, nel caso specifico, la teoria della giustizia e della carità $\mathrm{e}$, in generale, la teoria etica, più coerente e dunque che vi sia una soluzione corretta alla domanda "come dobbiamo comportarci in questa situazione?". Se questo non significa eliminare il conflitto è comunque un modo per annacquarlo.

In secondo luogo, la breve ricostruzione della concezione strumentale della ragion pratica delineata da Amaya sottostima il ruolo che la ragione può avere anche se non si accoglie una qualche versione del cognitivismo etico. Su questo punto è bene dire qualcosa di più.

La disputa tra la concezione strumentale e quella non strumentale della razionalità pratica è molto antica. Essa, ad esempio, è formulata in modo chiaro nel dialogo platonico Eutifrone. Il "dilemma

${ }^{21}$ N. MacCormick, Ragionamento giuridico e teoria del diritto (1978, II ed. 1994), trad. e introduzione di A. Schiavello, edizione italiana a cura di V. Villa, Giappichelli, Torino, 2001. Nel corso del tempo MacCormick ha indebolito il suo originario scetticismo humeano. Pur non potendo approfondire qui questo punto, ritengo che ai fini dell'argomento avanzato nel testo le differenze siano trascurabili. Cfr. N. MacCormick, Rhetoric and the Rule of Law. A Theory of Legal Reasoning, University Press, Oxford, 2005, in particolare pp. 1-11.

${ }^{22}$ A. Amaya, Diez tesis, p. 47. 
di Eutifrone", in breve, èil seguente: desideriamo ciò che desideriamo perché crediamo che ciò che desideriamo è buono e, dunque, degno di essere desiderato owero consideriamo qual cosa un bene, quindi degno

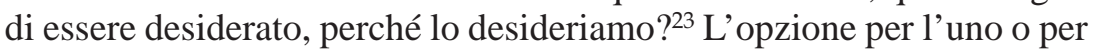
I'altro corno del di lemma-rispettivamente, quel lo cognitivista o quello non-cognitivista richiederebbe una discussi one articol ata e complessa, che in questa sede non occorre affrontare ${ }^{24}$. Ciò che mi interessa è mostrare che anche qual ora si propenda per la concezione debole della razionalità pratica -concezione che probabilmente è minoritaria nel di battito fil osofico contemporaneo ${ }^{25}$ - il risul tato non èquel lo di relegare la ragione e la razionalità in un angolo. A sostegno di quest' ultima affermazioneèsufficiente evidenziarequelli che sono i punti principali di intersezione o di incontro tra ragione e comportamento per la concezione strumental e della ragion pratica.

In prima battuta, come osserva anche Amaya, per la concezionestrumentale la ragione consente-ovviamente di distinguere tra comportamenti corretti escorretti, dati certi desideri. Se unapersona, ad esempio, desidera guarire da una mal attia ecerca di soddisfare questo desiderio affidandosi alle cure di un mago, sta ponendo in essere un comportamento sbagliato. Più precisamente, si tratta di un comportamento che, fatto salvo il suo desiderio di guarire, si fonda su una

\footnotetext{
${ }^{23}$ Riprendo la denominazione della controversia in questione come "dilemma di Eutifrone" ed anche le linee generali della formulazione di tale controversia da B. Celano, In difesa delle ragioni basate su desideri, "Ragion Pratica", 20, 2003, pp. 209-232.

${ }^{24}$ Non va peraltro scartata a priori la possibilità che si tratti di una questione indecidibile. Cfr., ad esempio, D. Lewis, Desire as Belief II, "Mind", New Series, Vol. 105. No. 418, 1996, pp. 303-313, in particolare pp. 305-307 e B. Celano, In difesa delle ragioni basate su desideri, cit., pp. 216-217, ove viene ripreso l'argomento di Lewis.

${ }^{25}$ Con riguardo al dibattito filosofico anglosassone, Giorgio Maniaci rileva che da circa un trentennio si assiste ad una reviviscenza del realismo morale e, dunque, di una concezione forte della razionalità pratica. Più in generale, l'autore nota come agli inizi del Novecento la prospettiva meta-etica dominante fosse l'intuizionismo, scalzata poi, intorno agli anni '30 e '40 del secolo scorso, dall'emotivismo. Cfr. G. Maniaci, Razionalità ed equilibrio riflessivo nell'argomentazione giudiziale, cit., p. 88.
} 
credenza falsa. Attraverso la ragione è possi bile svelare la falsità di questa credenza. Più in generale, la ragioneèin grado di indicare ex ante come bisogna comportarsi per soddisfare i propri desideri, e ex post consente di distinguere tra comportamenti corretti e scorretti (giusti e sbagliati, ecc.).

In seconda battuta, bisogna considerare che i desideri degli individui sono molteplici e tal vol ta contraddittori. Può succedere che una persona desi deri profondamente non contribuire ad aumentare la sofferenza nel mondo e, al tempo stesso, desideri mangiare un buon filetto al pepe verde per cena. Si tratta, evidentemente, di due desideri confliggenti, che è compito della ragione ordinare in modo seriale, tenendo conto dell' insiemetotal edei desi deri della persona in questione. Spetta dunqueall a ragione ancheil compito di organizzare in un sistema coerente i desideri degli individui. Ciò implica che la ragione esercita una influenza niente affatto trascurabile sul comportamento umano. Se ci si accorge che alcuni tra i propri desideri ne contraddicono altri, è possibile che certi desideri vengano espunti, anche sulla base di un test di coerenza, dal la schiera complessiva dei desi deri personali. Tornando all'esempio precedente, la consapevolezza della impossibilità di conciliare il desiderio di non incrementare la sofferenza del mondo e quello di mangiare un buon filetto al pepe verde, porterà la persona in questione a valutare il peso relativo dei due desideri (anche tenendo conto dell'insieme complessivo dei suoi desideri) e, qual ora stabilisse che il primo desiderio preval ga sul secondo, eviterà di mangiare carne per cena. Si può dire dunque che anche nel caso della concezione strumental e della ragione, la ragione èin grado di influire sui desideri26. Ciò non toglie, owviamente, che all'apice di ogni ragionamento sarà sempre possibile rinvenire un desiderio.

\footnotetext{
${ }^{26}$ Questo non significa - non necessariamente, almeno - che la ragione produca l'eliminazione o la scomparsa di determinati desideri. Il protagonista dell'esempio continuerà probabilmente a desiderare il suo filetto. Tuttavia, questo desiderio non produrrà alcuna azione perché l'azione conseguente, mangiare il filetto, è inibita da un altro desiderio, quello di non provocare sofferenza. È lecito ipotizzare, infine, che la consapevolezza delle sofferenze subite dagli animali di cui si cibano gli esseri umani possa produrre nell'individuo in questione la scomparsa del desiderio stesso di mangiare carne.
} 
In terza ed ultima battuta, si può plausibilmente ritenere che tra i desideri di un individuo viga una "gerarchia assiologica mobile"27. È possibile, in altri termini, che i desideri di ciascun essere umano non siano organizzati in una rigida scala gerarchica alla cui sommità si trovano alcuni desideri fondamentali, immuni sempre e comunque da qualsiasi tipo di critica razionale ${ }^{28}$. La gerarchia tra i desideri individuali potrebbe al contrario essere mobile, nel senso che potrebbe variare a seconda della concreta situazione di scelta che un essere umano si trova ad affrontare. In un caso, un determinato desiderio potrebbe essere trattato come fondamentale ed ultimo, costituendo così la base o il presupposto di un certo comportamento; in un'altra occasione, quello stesso desiderio potrebbe essere messo in questione, potrebbe essere fatto oggetto di una analisi al fine di valutare se effettivamente sussistano ragioni per agire sulla base di esso.

Quest'ultimo punto, insieme al precedente, consente di affermare che la tesi secondo la quale una concezione strumentale della razionalità pratica sarebbe incompatibile con l'elaborazione di una indagine etica normativa e, per quello che qui rileva, con un ragionamento coerentista è almeno discutibile. In parole più semplici, una obiezione che sovente viene mossa alla concezione strumentale della ragion pratica è quella di non essere in grado di dirci come dobbiamo comportarci in situazioni date: se il comportamento dipende dai desideri, allora qualsiasi comportamento è giustificato o giustificabile. Questa obiezione però è superficiale, in quanto trascura l'importante ruolo che è riconosciuto alla ragione anche dalla concezione strategica della razionalità pratica. In particolare, se l'indagine etica normativa viene svolta adottando un approccio coerentista che miri ad esempio al raggiungimento di un equilibrio riflessivo, allora tra la concezione non strumentale e la concezione strumentale della ragion pratica vi è la sola differenza che mentre nel primo caso ciò che si tenta di portare in equilibrio sono le credenze, nel secondo sono i desideri ${ }^{29}$.

\footnotetext{
${ }^{27}$ Riprendo liberamente questa espressione da R. Guastini, Teoria e dogmatica delle fonti, Giuffrè, Milano, 1998, pp. 302-304, ove essa viene utilizzata in relazione ai conflitti tra principi giuridici.

${ }^{28}$ Seguo qui B. Celano, In difesa delle ragioni basate su desideri, cit., pp. 222-225.

${ }^{29}$ Riprendo questa osservazione ancora da B. Celano, ult. cit., pp. 224-225, n. 24. 
Quel che mi preme sottolineare è che, alla luce di queste osservazioni, mi sembra almeno discutibile sostenere che vi sia incompatibilità tra ragionamento coerentista e concezione strumentale della ragion pratica.

Per concludere è opportuno aggiungere qualcosa su una concezione non strumentale della ragione e, in particolare, sull'oggettivismo etico.

L'oggettivismo etico sostiene a) che è possibile predicare la verità o la falsità degli enunciati che contengono valutazioni o apprezzamenti morali, b) che tali enunciati sono veri o falsi indipendentemente dalle nostre opinioni e c) che i canoni del ragionamento morale costituiscono un metodo affidabile per conseguire ed accrescere la conoscenza morale ${ }^{30}$. È importante sottolineare, in questa sede, che, all'interno di una concezione oggettivista dell'etica, e possibile distinguere una versione "monista" ed una versione "pluralista".

Il monismo etico sostiene che, per ogni situazione data, esiste un'unica soluzione giusta da un punto di vista morale. Il presupposto di questa posizione è che le ragioni per l'azione siano tra loro commensurabili e che gli eventuali conflitti tra tali ragioni in relazione ad una situazione data siano meramente apparenti, nel senso che esistono criteri oggettivi (ragioni per l'azione ulteriori) che consentono di stabilire oggettivamente quale tra $\mathrm{i}$ valori a prima vista confliggenti debba prevalere o quale compromesso possibile tra tali valori sia appropriato.

Il pluralismo o politeismo etico ${ }^{31}$ ritiene invece che i valori e gli ideali che popolano l'universo etico sono molteplici ed entrano

${ }^{30}$ Cfr. R. N. Boyd, How to Be a Moral Realist, in G. Sayre-McCord (ed. by), Essays on Moral Realism, Ithaca and London, Cornell University Press, 1988, pp. 181228, in particolare pp. 181-183.

${ }^{31} \mathrm{Su}$ questa prospettiva, cfr. B. Celano, Giusnaturalismo, positivismo giuridico e pluralismo etico, "Materiali per una storia della cultura giuridica" , 1, 2005, pp. 161-183; Id., Pluralismo etico, particolarismo e caratterizzazioni di desiderabilità: il modello triadico, "Ragion Pratica", 26, 2006, pp. 133-149. Cfr. altresì M. Barberis, L'eterogeneità del bene. Giuspositivismo, giusnaturalismo e pluralismo etico, in P. Comanducci e R. Guastini (a cura di), Analisi e diritto 2002-2003. Ricerche di giurisprudenza analitica, Torino, Giappichelli, 2004, pp. 1-20. 
inevitabilmente in conflitto tra loro. Come osserva Bruno Celano, «nulla garantisce che questi valori indichino, concordemente, un'unica direzione alle nostre scelte e alle nostre azioni: che essi, di volta in volta, vengano a comporre un tutto coerente, armonizzandosi gli uni con gli altri. Al contrario: gran parte delle scelte umane -alcune fra le scelte più significative- sono scelte tragiche, in situazioni nelle quali non si può far altro che sacrificare, in tutto o in parte, alcuni valori, in vista della promozione di altri (assumendosi, per di più, la responsabilità di tali scelte, e rassegnandosi all'ineluttabilità del rammarico)» ${ }^{32}$. Se si opta per l'oggettivismo etico politeista, la distanza tra concezione strumentale e concezione non strumentale della ragion pratica si assottiglia considerevolmente.

La ricostruzione un po' approssimativa di questioni molto complesse offerta in questo paragrafo può essere tollerata se commisurata all'obiettivo, assai modesto, che mi sono posto. Non ho inteso difendere una concezione strumentale della razionalità pratica rispetto ad una concezione non strumentale, ma soltanto avanzare qualche argomento a sostegno della compatibilità tra coerenza e noncognitivismo etico.

\section{Coerenza e inferenza Alla SPIEgAZIONE Migliore}

Contro l'argomento secondo cui la coerenza o, meglio, l'individuazione della soluzione più coerente, sia collegata all'intuizione e, dunque, non sia controllabile razionalmente, Amaya difende la tesi che l'inferenza coerentista è un tipo di inferenza esplicativa. Più precisamente, in Diez tesis si sostiene che l'inferenza coerentista ha l'obiettivo di individuare la spiegazione migliore, quella più coerente. In relazione al diritto, tre sono i passaggi che dovrebbero consentire di conseguire questo risultato. Il primo passaggio consiste nell'individuazione di tutti gli elementi rilevanti; con riguardo alla coerenza normativa tali elementi sono disposizioni normativi ed ipotesi interpretative, mentre con riguardo alla coerenza narrativa sono ipotesi fattuali, indizi o

${ }^{32}$ B. Celano, Giusnaturalismo, positivismo giuridico e pluralismo etico, cit., p. 175. 
elementi di prova. Il secondo passaggio richiede che si elaborino ipotesi alternative di decisione a prima vista plausibili. Il terzo ed ultimo passaggio consiste nella comparazione di tutte le ipotesi plausibili e nella successiva scelta della ipotesi più coerente e, dunque, giustificata. L'inferenza alla spiegazione migliore si distingue dunque dall'intuizione in quanto si tratta di un processo che può essere descritto ed in una certa misura controllato.

Da un lato, sono d'accordo con Amaya che la possibilità di controllare i vari passaggi dell'inferenza alla spiegazione migliore riduca la discrezionalità del decisore, al quale può essere richiesto di giustificare alcune scelte compiute nei diversi passaggi che compongono l'inferenza. Dall'altro, tuttavia, non credo che ciò basti a disinnescare l'obiezione secondo cui c'è un legame molto stretto tra coerenza ed intuizioni e/o preferenze irrazionali. Né il fatto che -sempre secondo Amaya- sia possibile formalizzare l'inferenza coerentista consente di superare questa obiezione. La stessa Amaya peraltro riconosce che «...estos formalismos logren la precisión, pero a costa de la complejidad y que, por lo tanto, sólo permitan dar cuenta de algunos de los aspectos del razonamiento coherentista» ${ }^{33}$.

Il problema, a mio avviso, non è tanto o soltanto la complessità, quanto le scelte valutative che si celano dietro ogni passaggio.

Il primo passaggio, come abbiamo visto, consiste nell'individuazione di tutti gli elementi rilevanti. Ma come si stabilisce se un elemento sia o meno rilevante?

Il secondo impone di elencare tutte le decisioni plausibili. Esistono criteri oggettivi per distinguere recisamente una decisione plausibile da una che non lo è?

Il terzo, infine, richiede di individuare la più coerente tra le decisioni plausibili. Si può ritenere che questa operazione possa essere compiuta facendo astrazione dalla propria concezione della giustizia, del diritto e dai propri pregiudizi?

In definitiva, opterei per una prospettiva più prudente in base alla quale l'inferenza alla spiegazione migliore ha un ruolo da giocare,

${ }_{33}$ A. Amaya, Diez tesis, p. 30. 
ma a partire da una serie di presupposti valutativi che inevitabilmente sfuggono ad un test di coerenza. Una spiegazione è migliore, dati certi presupposti. Questo significa che, in linea di principio, non esiste un'unica soluzione corretta (in quanto più coerente). La coerenza può contribuire a distinguere decisioni accettabili da decisioni inaccettabili; però non consente di dimostrare che una decisioni sia la migliore. Del resto, la stessa Amaya riconosce, a proposito della teoria di Paul Thagard del soddisfacimento di restrizioni che ella applica alla giustificazione giuridica, che un problema centrale che rimane senza soluzione è il modo in cui viene prodotto l'insieme degli elementi iniziali ritenuti rilevanti per comparare la coerenza di decisioni alternative. Ma è proprio questo il problema che impedisce di ritenere che esista una spiegazione migliore in assoluto.

Approfondiamo questo punto in relazione alla coerenza fattuale. La proposizione "è provato che $p$ " è l'ultimo tassello di un puzzle composto da molti elementi. Per usare la nota metafora della chain novel, un certo accadimento avvenuto nel passato (ad es.: Tizio ha ucciso la moglie) può essere ritenuto provato se è la conclusione più coerente di un ipotetico romanzo i cui capitoli precedenti sono rappresentati dagli elementi fattuali raccolti nel corso del processo ${ }^{34}$. L'idea è che sia sempre possibile stabilire quale sia il finale migliore di una storia. In alcuni casi, date le alternative a disposizione, è effettivamente così. Quando tutti gli indizi convergono verso una soluzione e non vi sono elementi a sostegno di una soluzione diversa si può ritenere che quella soluzione sia il tassello mancante del racconto processuale. Ad esempio, se a casa di Tizio viene ritrovata l'arma del delitto e alcuni indumenti di Tizio sporchi del sangue della moglie e, inoltre, nel corso del processo si appura che egli aveva stipulato un'assicurazione sulla vita della moglie, si può ritenere che la proposizione 'Tizio ha ucciso la moglie' sia più coerente con gli elementi a disposizione rispetto alla proposizione 'Tizio non ha ucciso la moglie'.

${ }^{34}$ Qui possiamo mettere tra parentesi le questioni inerenti al diverso grado di coerenza che una ipotesi fattuale deve avere in ambito civile e in ambito penale. Sui problemi specifici della prova dei fatti in ambito penale, cfr. J. Ferrer Beltrán, Prova e verità nel diritto, trad. it. V. Carnevale, Il Mulino, Bologna, 2004. 
Una decisione di condanna, in questo caso, è ampiamente giustificata, anche se, ovviamente, è sempre possibile che il marito non abbia ucciso la moglie e sia vittima di un complotto.

Basta tuttavia che la storia sia appena più complicata e gli elementi a disposizione un po' meno convergenti e la possibilità di individuare la conclusione migliore sulla base di un semplice test di coerenza non sembra plausibile. Di solito, i romanzi gialli ed i processi penali presentano una trama più articolata rispetto a quella, lineare, dell'esempio precedente. Anche in un caso apparentemente facile, come quello che ha visto sul banco degli imputati O. J. Simpson, vi sono elementi sufficienti per costruire storie diverse, tutte plausibili. Peraltro, in questo caso, non è stata prescelta dalla giuria la conclusione che, a mio avviso, era di gran lunga quella che si incastrava meglio con gli altri pezzi della storia, vale a dire, 'O. J. ha ucciso Nicole Brown, sua ex-moglie, e l'amico di lei, Ron Goldman' ma quella, a mio avviso ben più peregrina, che 'Nicole Brown e Ron Goldman sono stati uccisi da trafficanti di droga'.

In un interessante saggio volto ad investigare il ruolo della coerenza in questo caso giudiziale, Thagard sostiene che la decisione della giuria è l'esito di quella che egli denomina coerenza emotiva (emotional coherence), un misto di coerenza esplicativa e pregiudizi emotivi ${ }^{35}$. Ora, in questa sede non mi interessa approfondire questa nozione, né tanto meno discutere il sistema computazionale approntato da Thagard per render conto della coerenza emotiva. Mi limito solo a precisare che il piano del discorso di Thagard in quel saggio è esplicativo e non giustificativo e, dunque, non si pone il problema della "correttezza" della decisione della giuria ma delle "cause" di tale decisione e, dunque, della possibilità di predire tale decisione ${ }^{36}$. Quello che mi interessa rilevare è

${ }^{35} \mathrm{P}$. Thagard, Why wasn't O.J. convicted? Emotional coherence in legal inference, “Cognition and Emotion", 17 (3), 2003, pp. 361-383.

${ }^{36}$ Sulla distinzione tra "giustificazione" e "spiegazione", cfr. C. S. Nino, Legal Norms and Reasons for Action, "Rechtstheorie", 15, 1984, pp. 489-502, in particolare pp. 489-490; J. Raz, Reasons: explanatory and normative, in C. Sandis (edited by), New Essays in the Explanation of Action, Palgrave Macmillan, Basingstoke, 2009, pp. 184-202, in particolare pp. 186-189. Al riguardo si vedano anche le interessanti osservazioni sull'opportunità di "naturalizzare" la filosofia del diritto contenute nel par. 9 di Diez tesis. 
che la maggiore coerenza di una storia rispetto ad un'altra viene necessariamente valutata (anche) a partire da una serie di preferenze personali di vario genere che sfuggono ad una valutazione di tipo razionale. Ancora una volta, questo significa che non esiste la spiegazione migliore, ma molteplici spiegazioni accettabili, e che il prevalere dell'una rispetto alle altre dipende anche dai presupposti di partenza.

La perplessità sollevata in questo paragrafo presenta delle analogie rispetto alle obiezioni che vengono di solito avanzate nei confronti della tesi dworkiniana dell'unica risposta corretta. Ronald Dworkin replica a tali obiezioni sostenendo che chi rifiuta la one right answer thesis commette l'errore di ritenere che una proposizione (ad es.: X è la soluzione corretta del caso Y) che non può essere dimostrata come vera non può essere vera $o$, più correttamente, non può essere né vera né falsa. Secondo Dworkin, l'argomento dell'indimostrabilità -che presuppone l'accoglimento di una epistemologia rigidamente empirista e di una teoria della verità come corrispondenza- deve essere respinto per una serie di ragioni che qui non è necessario passare in rassegna. Supponiamo tuttavia che le ragioni addotte da Dworkin siano convincenti e, quindi, che per ogni caso giudiziale vi sia una risposta giusta (o, il che è lo stesso, per ogni questione giuridica e per ogni ricostruzione fattuale, esista la soluzione più coerente) anche se non è possibile dimostrare in modo conclusivo quale essa sia. La domanda da porsi è se postulare l'esistenza di una risposta corretta senza esplicitare i criteri conclusivi per la sua individuazione abbia una qualche rilevanza pratica (di tipo esplicativo e non retorico) all'interno di una teoria del ragionamento giuridico o di una teoria della prova ${ }^{37}$. Tutto quello che ho sostenuto sin qui fa propendere per una risposta di segno negativo.

\footnotetext{
${ }^{37}$ Cfr. S. R. Munzer, Right Answers, Preexisting Rights, and Fairness, "Georgia Law Review", Vol. 11, No. 5, 1977, pp. 1055-1068, in particolare p. 1063; E. Diciotti, Verità e certezza nell'interpretazione della legge, Giappichelli, Torino, 1999, pp. 214-218. Per un uso di più ampia portata dell'argomento dell'irrilevanza pratica, cfr. J. Waldron, The Irrelevance of Moral Objectivity, in R. P. George (edited by), Natural Law Theory. Contemporary Essays, Oxford University Press, Oxford, 1992, pp. 158-187.
} 


\section{Coerenza e responsabilità epistemicA}

In Diez tesis è possibile osservare una oscillazione che merita un approfondimento.

In un primo momento, vengono individuati e spiegati alcuni meccanismi che consentono di costruire la coerenza tra una serie di elementi. Ciò induce a ritenere che la coerenza non sia un dato da rilevare, ma un risultato da ottenere attraverso una serie di operazioni in senso lato interpretative e discrezionali. Per dirla con Dworkin, il diritto è un fenomeno interpretativo e l'attività conoscitiva posta in essere dai giuristi contribuisce alla determinazione dell'oggetto (del diritto, cioè). Per riprendere una felice metafora che Mario Jori usa per spiegare il metodo adottato da Scarpelli, «fare giurisprudenza non è come osservare un blocco di marmo, ma è come cantare in coro (...), perché il coro ci sia occorre che tutti o quasi i coristi cantino appunto in coro» ${ }^{38}$. In breve, lo studioso del diritto (giurista, filosofo del diritto...) non si colloca in una prospettiva esterna più o meno distaccata, ma è un partecipante impegnato a proporre -insieme e, il più delle volte, in competizione con i suoi colleghi- una interpretazione della pratica giuridica in the best light.

In un secondo momento, però, l'autrice si pone il problema di «(...) diferenciar entre la coherencia que produce justificación y la coherencia que es resultado de prejuicios» ${ }^{39}$. La distinzione in questione, se portata alle estreme conseguenze, sembra contraddire la tesi che la coerenza è il prodotto dell' attività interpretativa e non un dato inerte da rilevare.

Una eco di questa oscillazione si ritrova, ad esempio, in Tempo fuori luogo, romanzo di Philip K. Dick. Un personaggio del romanzo è un campione di un concorso a premi che consiste nel rispondere correttamente ad alcuni quesiti pubblicati quotidianamente sul giornale. In una occasione, parlando con il messo del giornale che gli consegnava gli assegni di una vincita, egli espresse la seguente considerazione: «Ho

\footnotetext{
${ }^{38}$ M. Jori, Uberto Scarpelli e il giuspositivismo, in U. Scarpelli, Cos'è il positivismo giuridico (1965), II Ed., Esi, Napoli, 1997, pp. 23-46, la citazione è a p. 26.

${ }^{39}$ A. Amaya, Diez tesis, p. 34.
} 
una teoria (...) non molto seria, a dire il vero, ma divertente. Magari non esiste una risposta giusta». Alla domanda del messo che, sorpreso, gli chiedeva su quali basi era allora possibile stabilire chi fosse il vincitore, egli rispose: «Magari date una scorsa alle soluzioni e scegliete quella che vi attira di più. Da un punto di vista estetico ${ }^{40}$.

Per approfondire la possibile contraddizione contenuta in Diez tesis, è bene osservare, innanzitutto, quali sono i processi che, secondo Amaya, consentono di costruire la coerenza nel (e del) diritto. L'autrice distingue analiticamente tre diverse tecniche.

La prima tecnica è la contrazione: un insieme incoerente di elementi può essere reso coerente eliminando l'elemento o gli elementi che producono dissonanza. Ad esempio, tutti gli elementi di prova fanno propendere per l'innocenza dell'imputato, tranne una testimonianza. La teoria dell'innocenza dell'imputato sarà rafforzata se si riesce ad espungere la testimonianza mettendo in dubbio la credibilità del testimone. La tecnica del distinguishing è una istanza di contrazione volta ad aumentare la coerenza di una ipotesi interpretativa.

La seconda è l'aggiunta: la coerenza di un insieme di elementi viene rafforzata aggiungendo elementi a sostegno di una determinata ipotesi interpretativa o fattuale. Tutto fa pensare che l'imputato sia colpevole, tranne una testimonianza che situa l'imputato lontano dalla scena del delitto. L'ipotesi della colpevolezza potrà essere resa più coerente attraverso l'aggiunta ad esempio della credenza che è alquanto improbabile che il testimone abbia potuto riconoscere l'imputato alla distanza ed alle condizioni di visibilità date.

L'ultima tecnica è la re-intepretazione: alcuni elementi vengono re-interpretati in modo tale da ridurre il grado di incoerenza dell'insieme. In relazione questa volta alla coerenza normativa, un insieme di precedenti giudiziali che vengono considerati inizialmente recalcitranti rispetto all'ipotesi interpretativa accolta, possono ad esempio essere resi coerenti con quest'ultima attraverso una reinterpretazione alla luce di un principio differente.

L'impressione di carattere generale è che tali tecniche non possano agire in vacuum, per così dire, ma presuppongano necessariamente una ipotesi (fattuale o interpretativa) che si vuole

${ }^{40}$ P. K. Dick, Tempo fuori luogo (1959), trad. it. G. Pannofino, Sellerio, Palermo, 1999, pp. 49-50. 
corroborare. È comunque l'ipotesi iniziale -che almeno in parte è il prodotto di intuizioni e pregiudizi- a spingerci ad attribuire un peso maggiore o minore a certi elementi. E questo va nella direzione della discrezionalità dei processi di coerentizzazione.

A sostegno dell'ampio margine di discrezionalità attribuito all'interprete si può sollevare qualche dubbio anche sulla distinzione tra questi tre meccanismi di costruzione della coerenza. A me pare, infatti, che, il più delle volte, la medesima operazione di coerentizzazione possa essere ricostruita ricorrendo indifferentemente a ciascuna di queste tecniche. Facciamo un esempio. A sostegno (della coerenza) dell'ipotesi che O. J. Simpson non abbia ucciso la ex-moglie e l'amico di quest'ultima, può essere addotto il fatto che i guanti sporchi di sangue ritrovati dalla polizia nel cortile di O. J. sono piccoli rispetto alle dimensioni delle mani dell'imputato. Che tecnica hanno usato gli avvocati di O. J. Simpson per accrescere il grado di coerenza dell'ipotesi a loro più favorevole? Si può sostenere che essi siano ricorsi alla contrazione: hanno eliminato un elemento di prova -i guanti sporchi di sangue- che contribuiva alla coerenza dell'ipotesi della colpevolezza del loro assistito. È tuttavia lecito ricostruire le cose in modo diverso ed affermare che la tecnica in gioco è l'aggiunta: gli avvocati hanno rafforzato la coerenza della loro ipotesi aggiungendo agli elementi a loro disposizione una serie di credenze, quali la credenza che i guanti sono troppo piccoli per essere stati usati da O. J., la credenza che sia stata la polizia a macchiare di sangue i guanti e a buttarli all'interno del cortile dell'imputato e così via. Infine, si può anche ricondurre l'attività degli avvocati alla tecnica della re-interpretazione: un elemento recalcitrante rispetto alla coerenza dell'ipotesi dell'innocenza dell'imputato viene reinterpretato in modo tale da essere compatibile con quell'ipotesi. I guanti sporchi di sangue ritrovati nella proprietà di O. J. sono compatibili con l'ipotesi dell'innocenza se la loro presenza viene ricondotta al tentativo della polizia di incastrare l'imputato.

Questa lunga disanima non è fine a se stessa, ma ha l'obiettivo, in parte già anticipato, di mostrare come la costruzione della coerenza sia altamente discrezionale. Non si tratta soltanto di mettere un tassello al posto giusto del puzzle, ma, per rimanere all'interno della metafora, di "costruire" i pezzi da cui il puzzle è composto. Per usare le parole di un 
titolo felice di un articolo di Luc Wintgens ${ }^{41}$, la coerenza coincide con la costruzione di un modo plausibile di raccontare una storia. E, rimescolando in un modo o nell'altro gli elementi a disposizione e variando la loro rilevanza, è spesso possibile raccontare più storie (egualmente) plausibili.

Come anticipato, Amaya ritiene che sia importante distinguere tra una coerenza in grado di produrre giustificazioni ed una coerenza che è il mero prodotto di nostri pregiudizi e che non ha alcun fondamento nella realtà. Se qualcuno vuole ricostruire la presunta coerenza di un sistema di norme a partire da un proprio principio morale che è antitetico rispetto ai principi fondamentali di quel sistema ovvero se si vuole suffragare una certa ipotesi fattuale (ad es.: O. J. Simpson ha ucciso la ex-moglie) a partire da certe credenze, come la tendenza a delinquere di certi gruppi sociali, dimostratesi false, allora la coerenza che si ottiene è il frutto di pregiudizi.

Se questa distinzione è intesa in senso debole, ritengo che essa sia perfettamente ammissibile: qualora un processo di coerentizzazione si fondi su credenze palesemente false o su presupposti chiaramente sbagliati, allora il risultato non è accettabile. Ci troviamo di fronte ad una giustificazione sbagliata. Come ho precisato nel secondo paragrafo, la coerenza consente di escludere un certo numero di soluzioni sbagliate, anche se, in linea di principio, non è in grado di individuare l'unica risposta giusta.

La mia impressione, tuttavia, è che Amaya intenda la distinzione in un senso più forte rispetto a quello appena tratteggiato. Una ipotesi fattuale o normativa è giustificata se è il risultato di un processo epistemicamente responsabile di massimizzazione della coerenza. Una ipotesi, in altri termini, è giustificata, se può essere ritenuta la migliore possibile da un decisore epistemicamente responsabile. Amaya distingue due diverse concezioni della responsabilità epistemica. Una concezione deontica, secondo la quale la responsabilità epistemica consiste in una serie di doveri specifici (il dovere di fondare le proprie credenze sulle prove, il dovere di ricercare

${ }^{41}$ L. J. Wintgens, A Plausibile Story and a Plausibile Way of Telling it. Some Comments on Dworkin's Philosophy of Law, "Rechtstheorie", 23, 1992, pp. 429444. 
prove su proposizioni la cui veridicità è dubbia, e così via). Vi è poi una concezione che considera la condotta epistemicamente responsabile come l'esito della pratica di certe virtù come la diligenza, l'imparzialità, l'apertura mentale e così via. Ovviamente, tra queste due concezioni possono esistere anche concezioni miste, alle quali va la preferenza di Amaya.

Da un lato, il sospetto è che la nozione di "responsabilità epistemica", quale che sia la concezione per la quale si opti, sia così vaga da svolgere un ruolo retorico a sostegno della decisione di volta in volta preferita da un decisore.

Una domanda che porrei ad Amaya è la seguente: la nozione di responsabilità epistemica consente ad esempio di escludere una delle due ipotesi alternative nel caso O. J. Simpson? È possibile sostenere la tesi che la giuria in quel caso non sia stata epistemicamente responsabile? Se la risposta a queste domande è negativa, allora credo che sarebbe meglio fare a meno di una nozione così filosoficamente densa (oltre che vaga).

Se si vuole sostenere che alcune ipotesi ricostruttive sono palesemente false e possono essere riconosciute come tali, allora non abbiamo bisogno della responsabilità epistemica. Ancora, se si attribuisce alla responsabilità epistemica lo stesso ruolo che Dworkin attribuisce al giudice Ercole, allora chi sposa una ipotesi piuttosto che un'altra potrà sempre dire che quell'ipotesi è la migliore in quanto è la decisione che sarebbe stata presa da un decisore epistemicamente responsabile. Come abbiamo detto nel paragrafo precedente, il problema dell'indimostrabilità evidenzia l'irrilevanza pratica (o, il che è lo stesso, la "retoricità") di alcune nozioni, filosoficamente molto attraenti, come "unica risposta giusta" e, per quel che qui rileva, "responsabilità epistemica".

Dall'altro lato, tuttavia, questa nozione sembra presupporre una qualche versione dell'oggettivismo meta-etico (monista). È come se si presupponesse l'esistenza dell'ipotesi più coerente e si attribuisse al decisore epistemicamente responsabile il compito di individuarla (non di costruirla). Dei problemi connessi ad una meta-etica oggettivista abbiamo accennato nel secondo paragrafo, e non è il caso di riprendere questo punto.

In conclusione, due mi sembrano le opzioni a disposizioni: o la nozione di responsabilità epistemica ricopre un ruolo retorico e, 
dunque, non rileva in relazione alla giustificazione di ipotesi fattuali e normative, ovvero è collegata ad una meta-etica oggettivista ed allora è una nozione incompatibile con la tesi che considera la coerenza qualcosa che si costruisce e non che si trova. 